\title{
Blunt Vascular Injuries in Patients with Cervical Spine Fractures: a Cohortclinical Study from Iran
}

\section{Samira Zabihyan, Humain Baharvahdat, Faraz Pirzeh, Yadollah Hosseini, Hamid Rezaee, Babak Ganjeifar*}

Department of Neurosurgery, Ghaem Hospital, Mashhad University of Medical Sciences, Mashhad, 99199-91766, Iran

\section{Introduction:}

Recent reviews have shown that almost $2.4 \%$ of patients with blunt cervical traumas suffer from fractures in the cervical vertebrae (Weber et al., 2018). The frequency of blunt traumas to cervical arteries varies in traumatic patients. The lack of alarming symptoms at the exhibition hinders proper diagnosis (Aizpuru et al., 2018). Computed tomography angiography (CTA) is accepted as the method of choice in many emergency departments for evaluating vascular injuries (VIs) in traumatic patients. Till date, a total of $98 \%$ concordance has been reported between digital Subtraction Angiography (DSA) and 16-slice CTA scanning (Tso et al., 2017). In addition to arterial involvement, CTA is an effective method for evaluation of the accompanying pathologies in the surrounding tissues (Laser et al., 2015). Blunt VIs have diverse clinical symptoms with poor presentations at admission. Reliable diagnostic technique is necessary for early detection and effective management of these traumas. This study aims to determine the frequency and clinical characteristics of carotid and vertebral artery injuries diagnosed by CTA in patients with cervical spine fractures secondary to blunt traumas.

\section{Case study:}

This cross-sectional clinical trial was conducted on adult patients with cervical spine fractures due to blunt traumas $(\mathrm{n}=106)$ admitted in Traumatic Emergency Center, Shahid Kamyab Hospital, Mashhad, Iran during May 2014 to April 2016. The main inclusion criterion was the definitive diagnosis of cervical trauma secondary to cervical spine fracture based on the findings from Computed tomography (CT) imaging accompanied by Magnetic Resonance Imaging (MRI). The exclusion criteria were pregnancy, a history of cervical tumors, cervical operations, radiotherapy, or penetrating cervical trauma.

CTA was conducted in patients with evidence of cervical spine fractures, using a standard device (Somatom Sensation 16 Protocol; Siemens, Germany). Bone injuries of
Study Area: Mashhad, Iran

Coordinates: $36^{\circ} 18^{\prime} \mathrm{N} ; 59^{\circ} 36^{\prime} \mathrm{E}$

Keywords: Cervical vertebrae, DSA, CTA, Blunt traumas.

Approved by the local Ethics Committee of Mashhad University of Medical Sciences (IR.MUMS.REC.1394.304).

the cervical spine and carotid and vertebral injuries were documented based on the imaging findings. Repeated CTA was performed in all patients with VIs after 7-10 days to evaluate any lesion progression. All the patients were followed-up (mean follow-up: 7.4 mon.; range, 6-12 mon.).

Although DSA is recognized as the gold standard for the diagnosis of VIs, we only used CTA in our study since its sensitivity and specif icity are close to DSA for diagnosis of injuries and follow-up of patients. Quantitative data are reported as mean \pm standard deviation (SD) (range), and qualitative data are presented as frequency.

The characteristics of patients with VIs are summarized in Table 1. The mean initial Glasgow Coma Scale (GCS) score was 13.12 \pm 2.89 (range of 7-15). Neurological deficits (33\%), Quadriparesis(15.1\%), Quadriplegia(9.4\%), Hemiparesis (5.7\%) and Hemiplegia (2.8\%) were reported in patients. Different types of cervical spine injuries were posterior element fracture (41.5\%), subaxial subluxation (20.7\%), subaxial burst fracture (18\%), odontoid fracture (Type-2: 6.6\%), Atlanto-axial subluxation $(3.9 \%)$, subaxial tear-drop fracture $(2.8 \%)$, Jefferson's fracture (2.8\%), Hangman's fracture $(2.8 \%)$, odontoid fracture (Type-3: 0.9\%).

Cervical arterial injury was detected in $6.6 \%$ patients, of them $1.9 \%$ showed carotid artery involvement and $4.7 \%$ with vertebral artery involvement. The carotid artery injury involved occlusion in one patient and dissection in the others (Fig.-1a,b,c). Moreover, vertebral artery injuries included occlusion in three patients (Fig.-2a,b,c) and

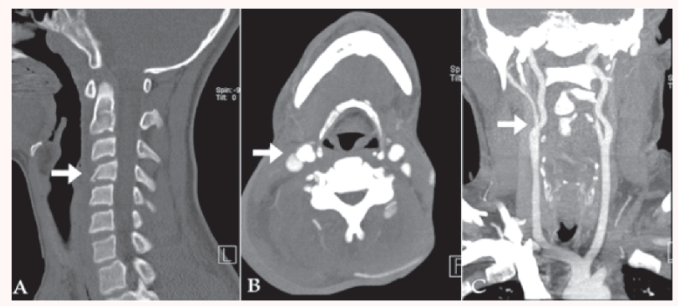

Figure-1: Burst fracture of fourth cervical vertebrae in a 33-year-old male (a) with dissection of the left internal carotid artery in axial (b) and coronal (c) computed tomography angiography 
Table 1.Characteristics of the patients with Vis (MVA- Motor Vehicle Accident; *Complication)

\begin{tabular}{llllllll}
\hline Arterial Injury & Sex & Age (y) & GCS & Cause & Neurological Report & Spinal Injury & Complication \\
\hline Dissection (Carotid) & Male & 33 & 15 & MVA & Quadriplegia & C4, burst fracture & - \\
Occlusion (Carotid) & Male & 54 & 11 & Fall & Hemiplegia & C5-6, Subluxation & Ischemia \\
Dissection (Vertebral) & Male & 24 & 7 & MVA & Quadriplegia & C3-4, Subluxation & - \\
Dissection (Vertebral) & Female & 32 & 9 & MVA & None & C2-C3, posterior element fracture & Ischemia \\
Occlusion (Vertebral) & Male & $\mathbf{1 8}$ & $\mathbf{1 5}$ & MVA & Quadriparesis & C4 posterior element fracture & - \\
Occlusion (Vertebral) & Male & $\mathbf{2 1}$ & 7 & MVA & None & C6 posterior element fracture & - \\
Occlusion (Vertebral) & Male & 41 & $\mathbf{1 5}$ & MVA & None & Jefferson's fracture & - \\
\hline
\end{tabular}

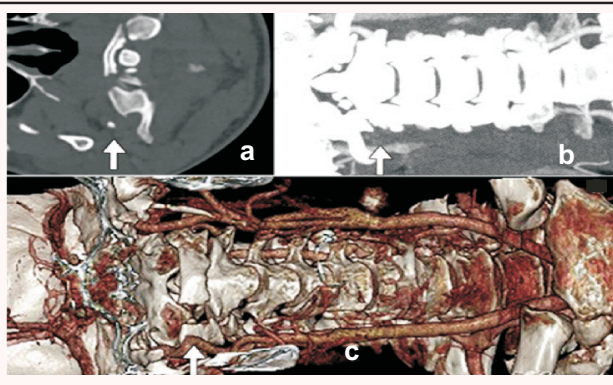

Figure-2: Jefferson's fracture in a 41-year-old male (a) with a resultant occlusion in the vertebral artery in the sagittal plane (b) $3 \mathrm{D}$ reformatted computed tomography angiography (c).

dissection in two cases. All the patients of VIs were treated with aspirin, with no complications during follow-up.

\section{Discussion:}

Incidence of arterial injuries in patients with cervical spine trauma ranges from $0.1 \%$ to $28.9 \%$ in different studies (Alterman et al., 2013). It has been shown that vertebral subluxations fractures of the upper cervical vertebrae and fractures of posterior elements with concomitant involvement of the foramen transversarium occur in over $90 \%$ of patients with cervical artery injuries (Nagasawa et al., 2017; Burks et al., 2018). The most common vertebral involvement in the present study was the fracture of posterior elements with the involvement of the foramen transversium, followed by subluxation and burst fractures. Differences in the incidence rate of arterial injuries could be attributed to the different methodologies and inclusion criteria with other studies. The incidence rate of carotid and vertebral injuries in patients with cervical spine traumas increases when concomitant cervical spine fractures are present (Alterman et al., 2013; Shahan et al., 2016). Although DSA is the gold standard for detecting injuries of cervical arteries, in practice, it cannot be applied in most traumatic cases due to its invasiveness (Aizpuru et al., 2018). Therefore, this modality has been replaced by other less invasive techniques, such as magnetic resonance angiography, duplex ultrasound, and CTA in most trauma centers. Although mild VIs are occasionally missed by CTA, most cases resolve spontaneously (Tso et al., 2017). The mortality rate was reported o-18\% in symptomatic patients (Mitha et al., 2013), while our finding showed 1.9\% of patients had brain ischemia and o\% mortality. Our finding showed the most common types of Arterial injuries were occlusion type (4 cases) and dissection types (3 cases) supporting the finding of Aizpuru et al. (2018).

Conclusively, the rate of cervical artery injuries in patients with cervical spine fractures was $6.6 \%$ in our series, which is higher than most of the previous reports. The higher incidence of these injuries in our study could be related to the selection of patients with cervical spine fractures. However, according to the findings, diagnostic investigation of VIs with CTA is necessary in patients with cervical spine fractures.

\section{References:}

Aizpuru, M., Poirier, M.V., Benarroch-Gampel, J. (2018): Vertebral artery injury: an update on screening, diagnosis and treatment. Current Surg. Rep., 6:22.

Alterman, D.M., Heidel, R.E., Daley, B.J., Grandas, O.H., Stevens, S.L., Goldman, M.H. \& Freeman, M.B. (2013): Contemporary outcomes of vertebral artery injury. J. Vasc. Surg., 57(3):741-746.

Burks, J.D., Conner, A.K., Briggs, R.G., Bonney, P.A., Smitherman, A.D., Baker, C.M., Glenn, C.A., Ghafil, C.A., Pryor, D.P., O'Connor, K.P. \& Bohnstedt, B.N. (2018): Blunt vertebral artery injury in occipital condyle fractures. I. Neurosurg. Spine, 29(5):500-505.

Laser, A., Kufera, J.A., Bruns, B.R., Sliker, C.W., Tesoriero, R.B., Scalea, T.M. \& Stein, D.M. (2015): Initial screening test for blunt cerebrovascular injury: validity assessment of wholebody computed tomography. Surgery, 158(3):627-635.

Mitha, A.P., Kalb, S., Ribas-Nijkerk, J.C., Solano, J., McDougall, C.G., Albuquerque, F.C., Spetzler, R.F. \& Theodore, N. (2013): Clinical outcome after vertebral artery injury following blunt cervical spine trauma. World Neurosurg., 8o(3-4):399-404.

Nagasawa, D.T., Bui, T.T., Lagman, C., Lee, S.J., Chung, L.K., Niu, T., Tucker, A., Gaonkar, B., Yang, I. \& Macyszyn, L. (2017): Isolated transverse process fractures: a systematic analysis. World Neurosurg.,100:336-341.

Shahan, C.P., Magnotti, L.J., Stickley, S.M., Weinberg, J.A., Hendrick, L.E., Uhlmann, R.A., Schroeppel, T.J., Hoit, D.A., Croce, M.A. \& Fabian, T.C. (2016): A safe and effective management strategy for blunt cerebrovascular injury: avoiding unnecessary anticoagulation and eliminating stroke. L. Trauma Acute CareSurg., 8o(6):915-922.

Tso, M.K., Lee, M.M., Ball, C.G., Morrish, W.F., Mitha, A.P., Kirkpatrick, A.W. \& Wong, J.H. (2017): Clinical utility of a screening protocol for blunt cerebrovascular injury using computed tomography angiography. I. Neurosurg., 126(4):1033-1041.

Weber, C.D., Lefering, R., Kobbe, P., Horst, K., Pishnamaz, M., Sellei, R.M., Hildebrand, F., Pape, H.C., TraumaRegister DGU. (2018): Blunt cerebrovascular artery injury and stroke in severely injured patients: an international multicenter analysis. World J. Surg., 42(7):2043-2053. 